\title{
Analytical Bootstrap Methods for Censored Data
}

ALAN D. HUTSON $\dagger$

Division of Biostatistics, P.O. Box 100212, University of Florida, Gainesville, FL 32610 USA

\begin{abstract}
Analytic bootstrap estimators for the moments of survival quantities are derived. By using these expressions recommendations can be made as to the appropriateness of bootstrap estimation under censored data conditions.
\end{abstract}

Keywords: Kaplan-Meier estimator, Monte Carlo resampling.

\section{Introduction}

It is well known that in the i.i.d. continuous univariate sample setting nonparametric bootstrapping consists of "plugging in" the empirical distribution function, $\hat{F}_{n}(x)=\sum_{i=1}^{n} I_{\left[x_{i} \leq x\right]} / n$, into some functional of interest, $\alpha(F)$, in order to obtain the corresponding bootstrap estimate $\alpha\left(\hat{F}_{n}\right)$. A simple example of this is the bootstrap estimate of $E(X)$, which is given by the sample mean $\bar{X}$ obtained by plugging in $\hat{F}_{n}$ for $F$ into $\alpha(F)=\int x d F$. However, it is much more difficult, yet not impossible, to directly obtain bootstrap estimates of quantities such as $\operatorname{Var}\left(\bar{X}^{2}\right)$. In practice, the standard approach to bootstrapping has been to estimate the quantity $\alpha\left(\hat{F}_{n}\right)$ with a Monte Carlo estimate $\widehat{\alpha\left(\hat{F}_{n}\right)}$ even if $\alpha\left(\hat{F}_{n}\right)$ is readily available. This is due in large part to the relative ease of programming the Monte Carlo method as compared to calculating $\alpha\left(\hat{F}_{n}\right)$. Therefore, most textbooks start with the Monte Carlo approach, oftentimes to the exclusion of the "exact" approach, e.g. see such popular bootstrapping texts as Efron and Tibshirani (1993), Shao and Tu (1995), and Davison and Hinckley (1997).

The classical Monte Carlo procedure involves resampling $B$ times from the data $\mathbf{X}=\left(X_{1}, X_{2}, \cdots, X_{n}\right)$ with replacement and then obtaining $B$ bootstrap replications, ultimately yielding the estimate $\widehat{\alpha\left(\hat{F}_{n}\right)}$ of the bootstrap estimator $\alpha\left(\hat{F}_{n}\right)$, a cruder estimate of $\alpha(F)$ due to simulation error on top of sampling error. For a proper bootstrap procedure the limit $\widehat{\alpha\left(\hat{F}_{n}\right)}$

$\dagger$ Requests for reprints should be sent to Alan D. Hutson, Division of Biostatistics, P.O. Box 100212, University of Florida, Gainesville, FL 32610 USA. 
should converge to $\alpha\left(\hat{F}_{n}\right)$, as the number of resamples, $B$, goes to infinity. Booth and Sarkar (1998) make a global recommendation for the choice of $B$ of around 800 replications. However, there are many components that factor into the convergence rates, such as the smoothness $\alpha\left(\hat{F}_{n}\right)$. In the age of high-speed computers $B$ can be taken much larger than 800 in the small to moderate sample size cases. Therefore, the question is why use the Monte Carlo approach when "exact" estimators such as those outlined in Hutson and Ernst (2000) and Huang (1991) are becoming more readily available and easily programmed. Some justifications are as follows:

1. Reproducibility. The value of $\alpha\left(\hat{F}_{n}\right)$ is "fixed" given the data.

2. Zero simulation error.

3. Mathematically attractive. The statistical properties of $\alpha\left(\hat{F}_{n}\right)$ are much easier to verify and study than those $\widehat{\alpha\left(\hat{F}_{n}\right)}$.

4. Contrary to popular belief there are occasions where the analytical approach is easier, e.g. see Hutson(1999) and Hutson(2001) for two meaningful examples.

5. The classical Monte Carlo approach may not provide a proper bootstrap procedure given censored data, i.e. in certain situations $\lim \widehat{\alpha\left(\hat{F}_{n}\right)} \neq$ $\alpha\left(\hat{F}_{n}\right)$, as $B \rightarrow \infty$.

Following from the last point given above we provide some useful and "exact" one and two-sample bootstrap quantities useful in survival analysis by making use of the well known product-limit estimator introduced by Kaplan and Meier (1958), with the empirical survival function estimator defined as

$$
\hat{S}_{n}(t)= \begin{cases}\prod_{T_{(j)} \leq t}\left(\frac{n-j}{n-j+1}\right)^{\delta_{(j)}}, & T<T_{(n)}, \\ 0, & t \geq T_{(n)},\end{cases}
$$

where $T_{(1)} \leq T_{(2)} \leq \cdots \leq T_{(n)}$ are the order statistics corresponding to the i.i.d. sample of $n$ failure or censoring times $T_{1}, T_{2}, \cdots, T_{n}$, and $\delta_{(1)}, \delta_{(2)}, \cdots, \delta_{(n)}$ are censoring indicators corresponding to the ordered $T_{i}$ 's, respectively. A value of $\delta_{(i)}=1$ indicates that $T_{(i)}$ is uncensored, while a value of $\delta_{(i)}=0$ indicates that $T_{(i)}$ is censored. To the best of our knowledge no analytical nonparametric bootstrap estimators for $\alpha(F)$ have been developed based upon the Kaplan-Meier estimator or any other nonparametric estimator, accounting for censored data.

The typical Monte Carlo approach for censored data is similar to the classical approach described above where the pairs $\left(T_{(i)}, \delta_{(i)}\right), i=1,2, \cdots, n$ 
are sampled with replacement with probability $1 / n$ assigned to each pair. The bootstrap quantities of interest are then obtained via information summarized over recalculations of the Kaplan-Meier estimator (or other similar estimators) given by each bootstrap replication, e.g. see Barber and Jennison (1999) and the references therein. This approach of resampling has some undesirable properties, which can be avoided through the "exact" approach. It should be clear that even though by definition $\hat{S}_{n}(t)$ from equation (1) is defined to be a proper estimator of the survivor function problems can arise when a given bootstrap resample has large proportion of censored observations. Should that particular bootstrap resample be used in the calculations or should it be tossed out?

\section{Moment Estimators of Survival Quantities}

The general approach for estimating the "exact" bootstrap moments of the functional $E(g(T))$, given censored data, is straightforward. We develop the expressions for important survival quantities such $E\left(T^{k}\right), E\left(\hat{S}\left(t_{0}\right)^{k}\right)$, $E\left(\left[\hat{F}^{-1}(u)\right]^{k}\right)$ and $E\left(\hat{F}^{-1}(u) \hat{F}^{-1}(v)\right)$, corresponding to the lifetime central moments, the survival fraction, the survival quantile moments, and the covariance between survival quantile moments, respectively. In addition, a kernel-quantile estimator of survival times falls out as a by-product, which corresponds to the kernel quantile estimator of Harrell and Davis (1982) when all observations are uncensored.

For an independent and identically distributed (i.i.d.) continuous sample of size $n$ from a distribution having positive support let $T_{i}=\min \left(X_{i}, C_{i}\right)$, where $X_{i}$ denotes a failure time and $C_{i}$ denotes a right censoring time. The value of $X_{i}$ is known only if $X_{i} \leq C_{i}$. In addition, let the indicator variable $\delta_{(i)}=1$ if $X_{i} \leq C_{i}$, and 0 otherwise, and let $X_{(1)} \leq X_{(2)} \leq \cdots \leq X_{(m)}$ denote the ordered observed failure times, $m \leq n$. Then we have the following:

Theorem 2.1. The exact $k$ th bootstrap moment about the origin for the random variable $T$ is given by

$$
E_{\hat{S}_{n}(T)}\left(T^{k}\right)=\left\{\begin{array}{lr}
\sum_{i=1}^{m} X_{(i)}^{k}\left(\hat{S}_{n}\left(X_{(i-1)}\right)-\hat{S}_{n}\left(X_{(i)}\right)\right), & \delta_{(n)}=1, \\
\sum_{i=1}^{m} X_{(i)}^{k}\left(\hat{S}_{n}\left(X_{(i-1)}\right)-\hat{S}_{n}\left(X_{(i)}\right)\right) & \\
+T_{(n)}^{k} \hat{S}_{n}\left(X_{(i)}\right), & \delta_{(n)}=0,
\end{array}\right.
$$

where $\hat{S}_{n}(T)$ is the Kaplan-Meier estimator defined at equation (1) and by definition $X_{(0)}=0$ implies $\hat{S}_{n}\left(X_{(0)}\right)=1$. 
Proof. For the case $\delta_{(n)}=1$, the expectation of $T^{k}$ is given by

$$
\begin{aligned}
E\left(T^{k}\right) & =\int_{0}^{\infty} t^{k} d F=\int_{0}^{1}\left[F^{-1}(u)\right]^{k} d u, \\
& =\sum_{i=1}^{m} \int_{1-\hat{S}_{n}\left(X_{(i-1)}\right)}^{1-\hat{S}_{n}\left(X_{(i)}\right)}\left[F^{-1}(u)\right]^{k} d u .
\end{aligned}
$$

Then the bootstrap estimator follows by substituting $\hat{F}_{n}=1-\hat{S}_{n}$ from equation (1) for $F^{-1}(u)$ into equation (3), where $\hat{F}_{n}^{-1}(u)$ is constant in the half-closed interval, $\left(1-\hat{S}_{n}\left(X_{(i-1)}\right), 1-\hat{S}_{n}\left(X_{(i)}\right)\right]$ and is equal to $X_{(i)}$. The case $\delta_{(n)}=0$ follows after slight modification.

It follows that the bootstrap mean and variance of $T$ are given by $\mu_{\hat{S}_{n}(T)}=$ $E_{\hat{S}_{n}(T)}(T)$ and $\sigma_{\hat{S}_{n}(T)}^{2}=E_{\hat{S}_{n}(T)}\left(T^{2}\right)-\left[E_{\hat{S}_{n}(T)}(T)\right]^{2}$, respectively. Therefore a naive two-sided bootstrap- $t$ confidence interval for expected lifetime, $E(T)$, is given by

$$
\mu_{\hat{S}_{n}(T)} \pm t_{n-1, \alpha / 2} \sigma_{\hat{S}_{n}(T)},
$$

where $t_{n-1, \alpha / 2}$ denotes the upper $\alpha / 2$ th percentile from a Student- $t$ distribution.

Define the quantile function estimator $\hat{F}^{-1}(u)=X_{(i)}$, such that $\hat{S}_{n}\left(X_{(i)}\right) \leq$ $1-u<\hat{S}_{n}\left(X_{(i-1)}\right), i=1,2, \cdots, m(\leq n)$. Then we have the following:

Theorem 2.2. The exact $k$ th bootstrap moment estimator for $\hat{F}^{-1}(u)$ for the case $\delta_{(n)}=1$ is given by

$$
E_{\hat{S}_{n}(T)}\left[\left(\hat{F}^{-1}(u)\right)^{k}\right]=\sum_{j=1}^{m} X_{(j)}^{k} w(j)_{p, q},
$$

where

$$
w(j)_{p, q}=\beta_{p, q}\left(1-\hat{S}_{n}\left(X_{(j)}\right)\right)-\beta_{p, q}\left(1-\hat{S}_{n}\left(X_{(j-1)}\right)\right),
$$

$\beta_{p, q}(\cdot)$ denotes the cumulative beta distribution with parameters $p=[n u]+$ 1 and $q=n-[n u]+2$, and $[\cdot]$ denotes the floor function. For the case $\delta_{(n)}=0$ add the term $T_{(n)}^{k} \beta_{p, q}\left(1-\hat{S}_{n}\left(X_{(j)}\right)\right)$ to equation (5).

Proof. The proof follows similarly to Theorem 2.1 with a simple substitution of $\hat{S}_{n}$ for $S$ into $E\left[\left(\hat{F}^{-1}(u)\right)^{k}\right]$, where $\hat{F}^{-1}(u)=Y_{([n u]+1)}$ denotes the $([n u]+1)$ st, possibly unobserved, order statistic from a sample of size $n$. 
A naive two-sided bootstrap- $t$ confidence interval for expected $u$ th survival quantile, $E_{\hat{S}_{n}(T)}\left[\hat{F}^{-1}(u)\right]$, is given by

$$
\mu_{\hat{F}^{-1}(u)} \pm t_{n-1, \alpha / 2} \sigma_{\hat{F}^{-1}(u)},
$$

where the parameters $\sigma_{\hat{F}^{-1}(u)}^{2}=E_{\hat{S}_{n}(T)}\left[\left(\hat{F}^{-1}(u)\right)^{2}\right]-\left[E_{\hat{S}_{n}(T)}\left(\hat{F}^{-1}(u)\right)\right]^{2}$ and $\mu_{\hat{F}^{-1}(u)}=E_{\hat{S}_{n}(T)}\left(\hat{F}^{-1}(u)\right)$. A more accurate confidence interval based upon exact bootstrap percentile distribution for $\hat{F}^{-1}(u)$ is provided in the next section.

As a by-product of Theorem 2.2, equation (5) can be modified to provide a kernel quantile function estimator, which reduces to the kernel quantile function estimator of Harrell and Davis (1982) when all observations are uncensored. Unlike the more traditional kernel quantile function estimators with symmetric kernel functions and having the form $\hat{F}_{k}^{-1}(u)=$ $h_{n}^{-1} \int_{0}^{1} F_{n}^{-1}(t) K\left((t-u) / h_{n}\right) d t$, the extension of the Harrell-Davis estimator has an asymmetric kernel, e.g. see Sheather and Marron (1990) for a general study of kernel quantile function estimators based upon uncensored observations and Padgett (1986) for the development of kernel quantile function estimators for censored data. The extension of the Harrell-Davis kernel quantile function estimator for censored observations is defined as

$$
\hat{F}_{k}^{-1}(u)=\sum_{j=1}^{m} X_{(j)}\left[\beta_{p, q}\left(1-\hat{S}_{n}\left(X_{(j)}\right)\right)-\beta_{p, q}\left(1-\hat{S}_{n}\left(X_{(j-1)}\right)\right)\right],
$$

where $\beta_{p, q}(\cdot)$ denotes the cumulative beta distribution with parameters $p=n u$ and $q=n(1-u), 0<u<1$. Xiang (1995) provides a theoretical examination of the strengths and weakness of these types of estimators.

Theorem 2.3. The exact bootstrap estimator of $\operatorname{Cov}\left(\hat{F}^{-1}(u), \hat{F}^{-1}(v)\right)$, $u<v, r=[n u]+1, s=[n v]+1$, for the case $\delta_{(n)}=1$ is given by $\operatorname{Cov}_{\hat{S}_{n}(T)}\left(\hat{F}^{-1}(u), \hat{F}^{-1}(v)\right)=\operatorname{Cov}(u, v)$, such that

$$
\begin{aligned}
\operatorname{Cov}(u, v) & =\sum_{j=2}^{m} \sum_{i=1}^{j-1} w_{i j(r s)}\left(X_{(i)}-\mu_{\hat{F}^{-1}(u)}\right)\left(X_{(j)}-\mu_{\hat{F}^{-1}(v)}\right) \\
& +\sum_{j=1}^{m} v_{j(r s)}\left(X_{(j)}-\mu_{\hat{F}^{-1}(u)}\right)\left(X_{(j)}-\mu_{\hat{F}^{-1}(v)}\right),
\end{aligned}
$$


where $\hat{\mu}_{(u)}$ is defined above, the weights are given by

$$
\begin{aligned}
w_{i j(r s)} & ={ }_{n} C_{r s} \sum_{k=0}^{s-r-1}\left(\begin{array}{c}
s-r-1 \\
k
\end{array}\right) \frac{(-1)^{s-r-1-k}}{s-k-1} \\
& \times\left[\hat{F}_{i}^{s-k-1}-\hat{F}_{i-1}^{s-k-1}\right]\left[B\left(\hat{F}_{j} ; k+1, n-s+1\right)\right. \\
& \left.-B\left(\hat{F}_{j-1} ; k+1, n-s+1\right)\right], \\
v_{j(r s)} & ={ }_{n} C_{r s} \sum_{k=0}^{s-r-1}\left(\begin{array}{c}
s-r-1 \\
k
\end{array}\right) \frac{(-1)^{s-r-1-k}}{s-k-1} \\
& \times\left\{B\left(\hat{F}_{j} ; s, n-s+1\right)-B\left(\hat{F}_{j-1} ; s, n-s+1\right)\right. \\
& -\left(\hat{F}_{j-1}\right)^{s-k-1}\left[B\left(\hat{F}_{j} ; k+1, n-s+1\right)\right. \\
& \left.\left.-B\left(\hat{F}_{j-1} ; k+1, n-s+1\right)\right]\right\},
\end{aligned}
$$

$\hat{F}_{i}=1-\hat{S}_{n}\left(X_{(i)}\right), B(x ; a, b)=\int_{0}^{x} t^{a-1}(1-t)^{b-1} d t$, and ${ }_{n} C_{r s}=n ! /[(r-$ $1) !(s-r-1) !(g-s)$ !]. If $\delta_{(n)}=0$ equation (8) can be modified similarly to the previous results by adding the terms in the summands corresponding to $T_{(n)}\left(>X_{(m)}\right)$.

Proof. Hutson and Ernst (2000) prove the specific case $m=n$. The results above follow similarly by replacing $\hat{F}_{n}(x)=\sum_{i=1}^{n} I_{\left[x_{i} \leq x\right]} / n$ with $\hat{F}_{n}(x)=1-\hat{S}_{n}(x)$, based upon the Kaplan-Meier estimator defined at (1), into equation (8).

If we let $Y_{(1)} \leq Y_{(2)} \leq \cdots \leq Y_{(n)}$ denote the order statistics of a sample of size $n$, possibly unobserved, we can modify equations (5) and (8) in order to obtain exact bootstrap mean and variance expressions for $\hat{S}_{n}\left(t_{0}\right)$. Towards that end first define the bootstrap moment estimators corresponding to the 
indicator function $I_{\left[Y_{(r)}\right] \leq t_{0}}$ and given by

$$
\begin{aligned}
p_{1, r} & =E_{\hat{S}_{n}(T)}\left(I_{\left[Y_{(r)} \leq t_{0}\right]}\right) \\
& =\sum_{j=1}^{m} I_{\left[X_{(j)} \leq t_{0}\right]}\left[\beta_{p, q}\left(1-\hat{S}_{n}\left(X_{(j)}\right)\right)-\beta_{p, q}\left(1-\hat{S}_{n}\left(X_{(j-1)}\right)\right)\right](11) \\
p_{2, r} & =E_{\hat{S}_{n}(T)}\left(I_{\left[X_{(r)} \leq t_{0}\right]}^{2}\right) \\
& =\sum_{j=1}^{m} I_{\left[X_{(j)} \leq t_{0}\right]}\left[\beta_{p, q}\left(1-\hat{S}_{n}\left(X_{(j)}\right)\right)-\beta_{p, q}\left(1-\hat{S}_{n}\left(X_{(j-1)}\right)\right)\right](12) \\
p_{r, s} & =E_{\hat{S}_{n}(T)}\left(I_{\left[X_{(r)} \leq t_{0}\right]} I_{\left[X_{(s)} \leq t_{0}\right]}\right) \\
& =\sum_{j=2}^{m} \sum_{i=1}^{j-1} w_{i j(r s)} I_{\left[X_{(i)} \leq t_{0}\right]} I_{\left[X_{(j)} \leq t_{0}\right]}+\sum_{j=1}^{m} v_{j(r s)} I_{\left[X_{(j)} \leq t_{0}\right]}
\end{aligned}
$$

$\beta_{p, q}(\cdot)$ denotes the cumulative beta distribution with parameters $p=r$ and $q=n-r+1$, and the weights $w_{i j(r s)}$ and $v_{j(r s)}$ are given by equations (9) and (10), respectively. Then we have the following:

Theorem 2.4. The first two exact bootstrap moment estimators of the random variable $\hat{S}_{n}\left(t_{0}\right)$ for $\delta_{(n)}=1$ are given by

$$
\begin{aligned}
E_{\hat{S}_{n}(T)}\left[\hat{S}_{n}\left(t_{0}\right)\right] & =1-\sum_{r=1}^{m} p_{1, r} \\
E_{\hat{S}_{n}(T)}\left[\hat{S}_{n}\left(t_{0}\right)^{2}\right] & =1-2 \sum_{r=1}^{m} p_{1, r}+\sum_{r=1}^{m} p_{2, r}+2 \sum_{r=1}^{m} \sum_{s=1}^{m} I_{[r \neq s]} p_{r, s}
\end{aligned}
$$

where $p_{1, r}, p_{2, r}$ and $p_{r, s}$ are given above by equations (11), (12) and (13), respectively. It then follows that

$$
\operatorname{Var}_{\hat{S}_{n}(T)}\left[\hat{S}_{n}\left(t_{0}\right)\right]=E_{\hat{S}_{n}(T)}\left[\hat{S}_{n}\left(t_{0}\right)^{2}\right]-E_{\hat{S}_{n}(T)}\left[\hat{S}_{n}\left(t_{0}\right)\right]^{2} .
$$

Higher moments are possible, but are analytically difficult. If $\delta_{(n)}=0$ additional terms in the summands of equations (11), (12) and (13) corresponding to $T_{(n)}\left(>X_{(m)}\right)$ are needed.

Proof. The proof follows the same line of reasoning as the earlier proofs by noting that if we re-express the Kaplan-Meier estimator in the form $\sum_{i=1}^{n} I_{\left[Y_{(i)}\right] \leq t_{0}}$ then its moments are just weighted functions of the ordered failure times and is a constant function in the interval $\left(X_{(i-1)}, X_{(i)}\right]$. The integrations of the components then reduce to equations (11), (12) and (13). 
Similar to above, a naive two-sided bootstrap- $t$ confidence interval for expected survival probability, $E\left(\hat{S}\left(t_{0}\right)\right)$, is given by

$$
E_{\hat{S}_{n}(T)}\left[\hat{S}_{n}\left(t_{0}\right)\right] \pm t_{n-1, \alpha / 2} \sqrt{\operatorname{Var}_{\hat{S}_{n}(T)}\left[\hat{S}_{n}\left(t_{0}\right)\right]}
$$

where $t_{n-1, \alpha / 2}$ denotes the upper $\alpha / 2$ th percentile from a Student- $t$ distribution.

\section{One-sample and Two-sample Percentile Confidence Intervals for Survival Quantiles}

The classical approach for calculating nonparametric confidence intervals for $\alpha(F)$ in the univariate setting consists of drawing samples of size $n$ from the empirical distribution function $\hat{F}_{n}(x)$ and then calculating $\alpha\left(\hat{F}_{n}\right)$ $B$ times. The values of $\alpha\left(\hat{F}_{n}\right)$ are then ordered. The corresponding bootstrap $(1-\alpha) \times 100 \%$ percentile confidence interval for $\alpha(F)$ is then given by the interval corresponding to the $[B \alpha / 2]$ and $[B(1-\alpha / 2)]$ ordered values, where [.] denotes the floor function, e.g. see Diciccio and Efron (1996) for a discussion of this method. Recently, Polansky (1999) has studied the undercoverage of this method for small samples and provides bounds on the true coverage of the interval. Again, note that the Monte Carlo resampling approach to this problem is an approximation to the bootstrap estimated confidence interval and hence may add to the problem of undercoverage. Hutson and Ernst (1998) have shown that percentile intervals for $L$-estimators can be calculated directly by considering the functional

$$
\inf _{\theta \in \mathcal{R}} E\left\{\frac{\left|T_{n}-\theta\right|+(2 \alpha-1)\left(T_{n}-\theta\right)}{2}-\frac{\left|T_{n}\right|+(2 \alpha-1) T_{n}}{2}\right\},
$$

thus eliminating the simulation error and improving upon the coverage. The properties of (1) are outlined in Abdous and Theodorescu (1992) who generalize the definition of the $\alpha$-quantile to $\mathcal{R}^{k}$ space, $k \geq 1$.

In this section we illustrate how equation (1) can be modified in order to derive confidence intervals for a single quantile or differences between quantiles from two groups. The primary purpose is to develop a confidence interval for median differences in the presence of censored observations. As above, define the quantile function estimator $\hat{F}^{-1}(u)=X_{(i)}$, such that $\hat{S}_{n}\left(X_{(i)}\right) \leq 1-u<\hat{S}_{n}\left(X_{(i-1)}\right), i=1,2, \cdots, m(\leq n)$, then for $\delta_{(n)}=1$, the exact $(1-\alpha) \times 100 \%$ percentile confidence interval for the quantile $F^{-1}(u)$ 
is given by the values satisfying

$$
\begin{gathered}
\inf _{\theta \in \mathcal{R}}\left\{\sum_{j=1}^{m}\left[\left|X_{(j)}-\theta\right|+\left(2 \alpha^{\prime}-1\right)\left(X_{(j)}-\theta\right)\right] \times\right. \\
\left.\left[\beta_{p, q}\left(1-\hat{S}_{n}\left(X_{(j)}\right)\right)-\beta_{p, q}\left(1-\hat{S}_{n}\left(X_{(j-1)}\right)\right)\right]\right\},
\end{gathered}
$$

for $\alpha^{\prime}=\alpha / 2$ and $\alpha^{\prime}=1-\alpha / 2$, where $\mu_{\hat{F}^{-1}(u)}=E_{\hat{S}_{n}(T)}\left(\hat{F}^{-1}(u)\right)$ is defined at equation (5), $\beta_{p, q}(\cdot)$ denotes the cumulative beta distribution with parameters $p=i$ and $q=m-i+1$. The proof follows the lines of Theorem 2.2. For the case $\delta_{(n)}=0$ we need the additional term $\left[\mid T_{(n)}-\right.$ $\left.\theta \mid+\left(2 \alpha^{\prime}-1\right)\left(T_{(n)}-\theta\right)\right] \beta_{p, q}\left(1-\hat{S}_{n}\left(X_{(j)}\right)\right)$ in the summands above.

Alternative approaches for calculating nonparametric confidence intervals for quantiles, and more specifically the median, are proposed by Thomas and Grunkemeier (1975), Brookmeyer and Crowley (1982), Emerson (1982), Simon and Lee (1982), Wang and Hettmansperger (1990), Efron (1981) and Reid (1981). However, none of these approachs generalizes easily to the two-sample case. Kim (1993) has published a Monte Carlo algorithm for the two-sample case and examined the theoretical properties. Alternatively, equation (1) can be easily modified to provide nonparametric bootstrap for the quantities $F^{-1}(u)-G^{-1}(v)$, where $F^{-1}(u)$ and $G^{-1}(v)$ correspond to the quantiles from two independent populations, respectively.

Let $X_{(1)} \leq X_{(2)} \leq \cdots \leq X_{\left(m_{1}\right)}$ and $Y_{(1)} \leq Y_{(2)} \leq \cdots \leq Y_{\left(m_{2}\right)}$ denote the ordered observed failure times, with corresponding quantile function estimators $\hat{F}^{-1}(u)=X_{(i)}, \hat{S}_{m_{1}}\left(X_{(i)}\right) \leq 1-u<\hat{S}_{m_{1}}\left(X_{(i-1)}\right)$, and $\hat{G}^{-1}(v)=$ $Y_{(j)}, \hat{S}_{m_{2}}\left(Y_{(j)}\right) \leq 1-v<\hat{S}_{m_{2}}\left(Y_{(j-1)}\right)$, with $m_{1} \leq n_{1}$ and $m_{2} \leq n_{2}$, with the total sample size $N=n_{1}+n_{2}$. Then the exact $(1-\alpha) \times 100 \%$ percentile confidence interval for the quantile difference $F^{-1}(u)-G^{-1}(v)$ is given by the values satisfying

$$
\begin{aligned}
& \inf _{\theta \in \mathcal{R}}\left\{\sum_{k=1}^{m_{1}} \sum_{l=1}^{m_{2}}\left[\left|X_{(k)}-Y_{(l)}-\theta\right|+\left(2 \alpha^{\prime}-1\right)\left(X_{(k)}-Y_{(l)}-\theta\right)\right]\right. \\
\times & {\left[\beta_{p_{i}, q_{i}}\left(1-\hat{S}_{m_{1}}\left(X_{(k)}\right)\right)-\beta_{p_{i}, q_{i}}\left(1-\hat{S}_{m_{1}}\left(X_{(k-1)}\right)\right)\right] } \\
\times & {\left.\left[\beta_{p_{j}, q_{j}}\left(1-\hat{S}_{m_{2}}\left(Y_{(l)}\right)\right)-\beta_{p_{j}, q_{j}}\left(1-\hat{S}_{m_{2}}\left(Y_{(l-1)}\right)\right)\right]\right\}, }
\end{aligned}
$$

for $\alpha^{\prime}=\alpha / 2$ and $\alpha^{\prime}=1-\alpha / 2$, where $\mu_{\hat{F}^{-1}(u)}$ and $\mu_{\hat{G}^{-1}(v)}$ are defined for $X$ and $Y$ by equation (5), $\beta_{p_{i}, q_{i}}(\cdot)$ denotes the cumulative beta distribution with parameters $p_{i}=i$ and $q_{i}=m_{1}-i+1$, and $\beta_{p_{j}, q_{j}}(\cdot)$ denotes the cumulative beta distribution with parameters $p_{j}=j$ and $q_{j}=m_{2}-j+1$. 
Similar to the one-sample case, one or two additional terms need to be added to the summands above corresponding to whether or not the last observation in either or both samples is censored, respectively. Note that higher order contrasts from $k$-samples can be examined similarly.

\section{Simulation Study}

We reran the simulation experiment published in Brookmeyer and Crowley (1982) in order to compare median confidence intervals obtained by their method implemented in SAS Version 6.08 and higher with the new nonparametric method obtained as a minimization problem defined in the previous section, as well as with the new parametric method based on a Weibull model, labelled "NM" and "PM", respectively. The Brookmeyer and Crowley method is labelled "BC." The calculations of the new intervals are straightforward using software that currently exists. For this simulation study we employed the Weibull distribution given by the quantile function $Q(u)=(-\log (1-u))^{\theta}$ for arbitrary values of $\theta=1 / 2,1,5$. The censoring distribution was assumed to be uniform $(0, T)$. Each simulation consisted of 1000 replications of size $n=25$ at level $\alpha=0.01,0.05,0.10$ and values of $T=2,5,10$. The values of $T=2,5,10$ correspond to heavy, medium and light censoring, respectively. Table 4.1 contains the estimated coverage probabilities for the three methods. It is apparent from Table 4.1 that the new nonparametric (NM) based confidence intervals is comparable to the $\mathrm{BC}$ method. What is surprising however is that both nonparametric methods outperform the parametric intervals (PM) in the case of heavy censoring. The results given in Table 4.1 hold across a variety of distributions, samples sizes and censoring proportions. 
Table 4.1. Observed coverage probabilities $(n=25)$

\begin{tabular}{|c|c|c|c|c|c|c|c|c|c|c|}
\hline \multirow{3}{*}{ Method } & & \multicolumn{4}{|c|}{$\theta=1$} & \multicolumn{3}{c|}{$\theta=1 / 2$} & \multicolumn{3}{c|}{$\theta=5$} \\
\cline { 3 - 11 } & $T$ & \multicolumn{9}{|c|}{$\theta$-level } \\
\cline { 3 - 11 } NM & 0.01 & 0.05 & 0.10 & 0.01 & 0.05 & 0.10 & 0.01 & 0.05 & 0.10 \\
\hline & 5 & .96 & .89 & .80 & .89 & .80 & .67 & .99 & .96 & .89 \\
& 10 & .98 & .96 & .87 & .97 & .93 & .84 & .99 & .95 & .89 \\
\hline \multirow{3}{*}{$\mathrm{PM}$} & 2 & .94 & .79 & .68 & .90 & .73 & .61 & .97 & .88 & .79 \\
& 5 & .99 & .96 & .91 & .99 & .95 & .90 & .99 & .93 & .87 \\
& 10 & .99 & .97 & .93 & .99 & .96 & .93 & .99 & .94 & .91 \\
\hline \multirow{3}{*}{$\mathrm{BC}$} & 2 & .95 & .89 & .82 & .90 & .79 & .70 & .98 & .94 & .88 \\
& 5 & .98 & .94 & .87 & .97 & .92 & .85 & .98 & .94 & .88 \\
& 10 & .98 & .95 & .89 & .98 & .94 & .88 & .98 & .94 & .87 \\
\hline
\end{tabular}

The same simulation experiment was repeated for the two-sample case. The results in Table 4.2 look promising in terms of coverage probabilities. The utility of this method will be towards comparing two or more samples with respect to their given quantiles, e.g. no generally accepted nonparametric method currently exists for carrying out a comparison of the difference between medians given randomly right-censored observations.

Table 4.2. Observed two-sample coverage $\operatorname{probabilities}(n=25$ per group $)$

\begin{tabular}{|c|c|c|c|c|c|c|c|c|c|c|}
\hline \multirow[b]{3}{*}{ Method } & \multirow[b]{3}{*}{$T$} & \multicolumn{3}{|c|}{$\theta=1$} & \multicolumn{3}{|c|}{$\theta=1 / 2$} & \multicolumn{3}{|c|}{$\theta=5$} \\
\hline & & \multicolumn{9}{|c|}{$\alpha$-level } \\
\hline & & 0.01 & 0.05 & 0.10 & 0.01 & 0.05 & 0.10 & 0.01 & 0.05 & 0.10 \\
\hline \multirow{3}{*}{ NM } & 2 & .99 & .97 & .91 & .99 & .96 & .93 & .99 & .98 & .93 \\
\hline & 5 & .99 & .98 & .93 & .99 & .97 & .93 & .99 & .98 & .92 \\
\hline & 10 & .99 & .97 & .94 & .99 & .96 & .94 & .99 & .96 & .93 \\
\hline
\end{tabular}

\section{Acknowledgements}

The author is thankful to the editor, associate editor, and two referees for their careful review and helpful suggestions. Alan Hutson's work was supported in part by NIH General Clinical Research Center Grant RR0082.

\section{References}

1. Abdous, B. and Theodorescu, R. (1992), Note on the Spatial Quantile of a Random Vector, Statistics \& Probability Letters, 13, 333-336. 
2. Barber, S. and Jennison, C. (1999), Symmetric Tests and Confidence Intervals for Survival Probabilities and Quantiles of Censored Survival Data, Biometrics, 55, 430-436.

3. Booth J. G. and Sarkar S. (1998), Monte Carlo Approximation of Bootstrap Variances, The American Statistician, 52, 354-357.

4. Brookmeyer, R. and Crowley, J. (1982), A Confidence Interval for Median Survival Time, Biometrics, 38, 29-41.

5. Davison, A. C. and Hinkley, D. V. (1997), Bootstrap Methods and Their Application, Cambridge: Cambridge University Press.

6. Diciccio, T. J. and Efron, B. (1996), Bootstrap Confidence Intervals, Statistical Science, 11, 189-212.

7. Efron, B. (1981), Censored Data and the Bootstrap. Journal of the American Statistical Association, 76, 312-319.

8. Efron, B. and Tibshirani, R. (1993), An Introduction to the Bootstrap, New York: Chapman \& Hall.

9. Emerson, J. (1982), Nonparametric Confidence Intervals for the Median in the Presence of Right Censoring, Biometrics, 38, 17-27.

10. Harrell, F. E. and Davis, C. E. (1982), A New Distribution Free Quantile Estimator, Biometrika, 69, 635-640.

11. Huang, J. S. (1991), Efficient Computation of the Performance of Bootstrap and Jackknife Estimators of the Variance of L-Statistics, Journal of Statistical Computation and Simulation, 38, 45-56.

12. Hutson, A. D. and Ernst, M. D. (1998), An Exact Method For Calculating the Bootstrap Percentiles of a Functional of Order Statistics, Technical Report 582, Department of Statistics, University of Florida.

13. Hutson, A. D. (1999), Calculating Nonparametric Confidence Intervals for Quantiles Using Fractional Order Statistics, Journal of Applied Statistics, 26, 343-354.

14. Hutson, A. D. (2001), "Exact" Bootstrap Confidence Bands for the Quantile Function via Steck's Determinant, Journal of Computational and Graphical Statistics. (in press)

15. Hutson, A. D. and Ernst, M. D. (2000), The Exact Bootstrap Mean and Variance of an L-estimator, Journal of the Royal Statistical Society-Series B, 62, 89-94.

16. Kaplan, E. L. and Meier, P. (1958), Nonparametric estimation from incomplete observations, Journal of the American Statistical Association, 52, 457-481.

17. Kim, J. (1993), Two-sample Inference for Quantiles Based on the Bootstrap for Censored Survival Data, Journal of the Korean Statistical Society, 22, 159-169.

18. Padgett, W. J. (1986), A Kernel-Type Estimator of a Quantile Function from Right-Censored Data, Journal of the American Statistical Association, 81, 215222 .

19. Polansky, A. M. (1999), Upper bounds on the true coverage of bootstrap percentile type confidence intervals, The American Statistician, 53, 362-369.

20. Reid, N. (1981), Estimating the Median Survival Time. Biometrika, 68, 601-608.

21. Shao, J. and Tu, D. (1995), The Jackknife and Bootstrap, New York: SpringerVerlag.

22. Sheather, S. J. and Marron, J. S. (1990), Kernel Quantile Estimators, Journal of the American Statistical Association, 85, 410-416.

23. Simon, R. and Lee, Y. J. (1982), Nonparametric Confidence Limits for Survival Probabilities and Median Survival Time, Cancer Treatment Reports, 66, 37-42.

24. Thomas, D. R. and Grunkemeir, G. L. (1975), Confidence Interval Estimation of Survival Probabilities for Censored Data, The Journal of the American Statistical Society, 70, 865-871. 
25. Wang, J. L. and Hettmansperger, T. P. (1990), Two-sample Inference for Median Survival Times Based on One-Sample Procedures for Censored Survival Data, The Journal of the American Statistical Society, 85, 529-536.

26. Xiang, X. (1995), Deficiency of the Sample Quantile Function Estimator with Respect to Kernel Quantile Estimators for Censored Data, The Annals of Statistics, 23, 836-854. 


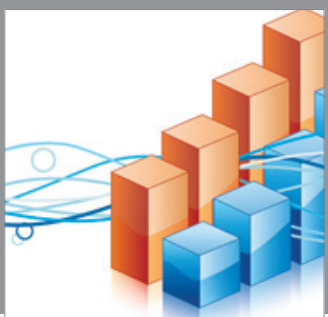

Advances in

Operations Research

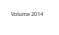

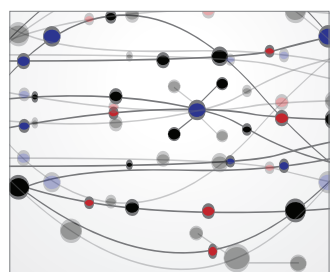

\section{The Scientific} World Journal
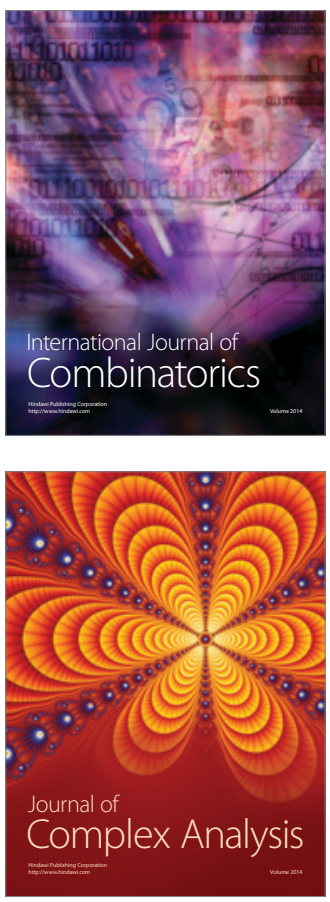

International Journal of

Mathematics and

Mathematical

Sciences
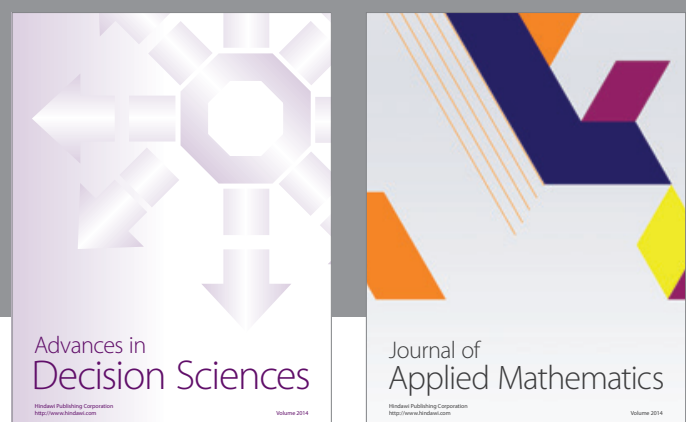

Journal of

Applied Mathematics
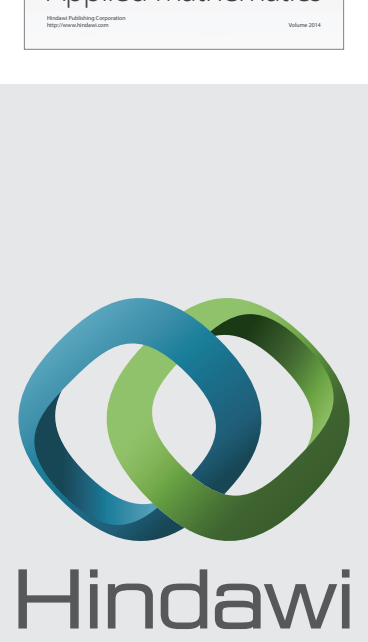

Submit your manuscripts at http://www.hindawi.com
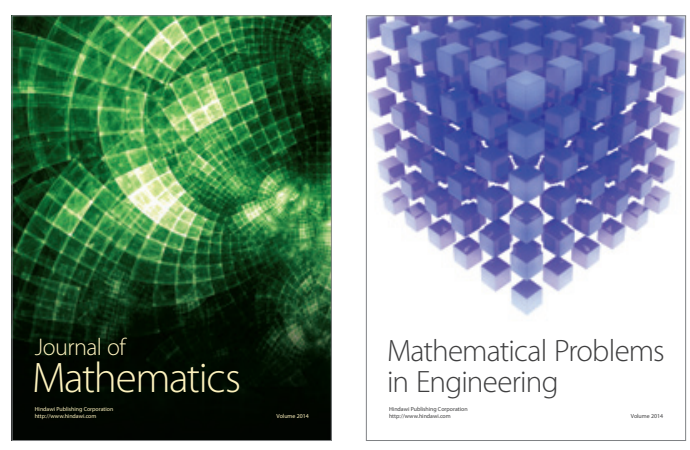

Mathematical Problems in Engineering
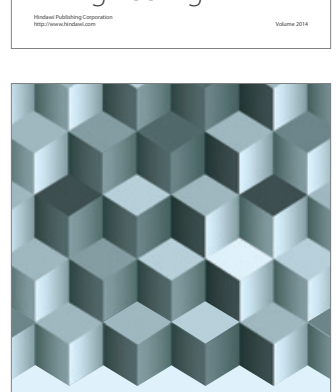

Journal of

Function Spaces
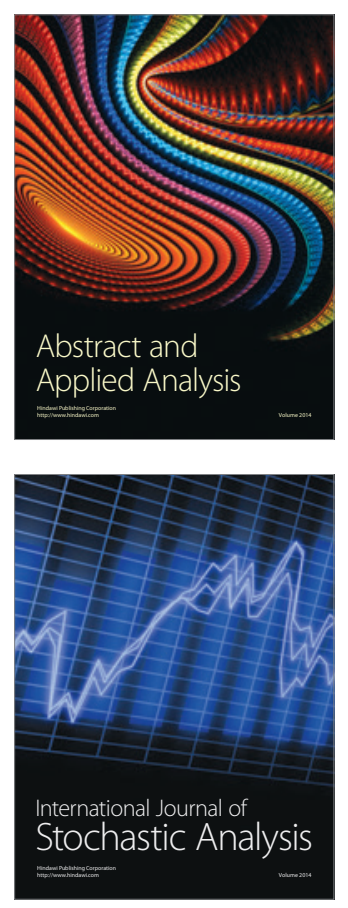

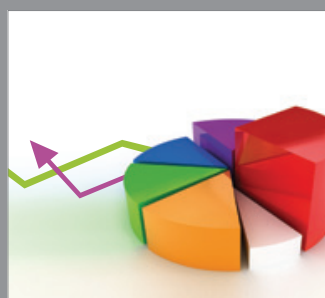

ournal of

Probability and Statistics

Promensencen
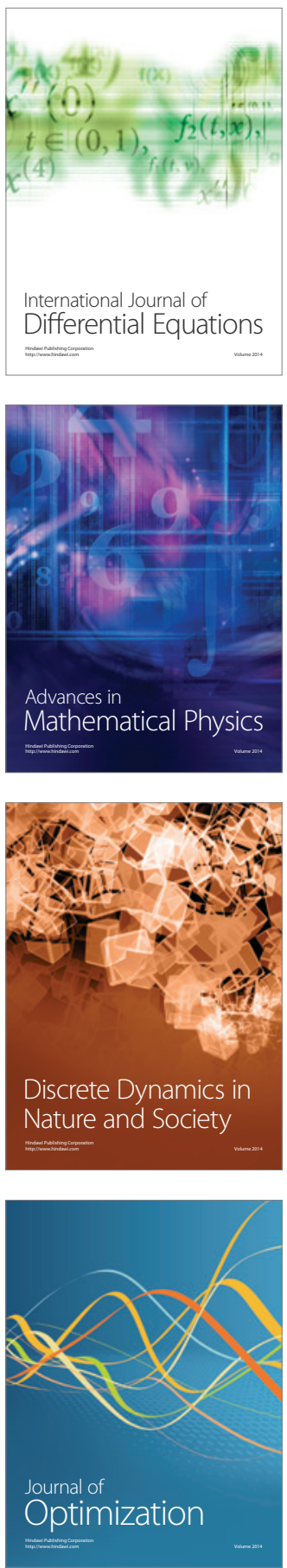